bination aus Emtricitabin-Tenofovir 200/300 mg einmal täglich und Lopinavir-Ritonavir 200/50 mg 2x2 Tabletten täglich über 36 Wochen. Bei allen übrigen Patienten wurde diese oder eine andere potente ART nach Maßgabe des behandelnden Arztes erst begonnen, wenn die Kriterien der klinischen Indikation vorlagen.

Bezüglich des primären Endpunkts wies die Gruppe mit der frühzeitigen Therapie in Woche 72 ein signifikant besseres Ergebnis auf als die Gruppe mit der aufgeschobenen Therapie in Woche 72 und in Woche 36. Der Unterschied bezog sich hauptsächlich auf eine ungünstige Entwicklung der immunologischen Parameter. Die Indikation für eine ART wurde von 50\% der Patienten mit der aufgeschobenen Therapie, jedoch nur von $10 \%$ der frühzeitig therapierten Patienten erreicht. Das Data Safety Monitoring Board empfahl aufgrund dieses Ergebnisses einen Abbruch der Studie, da die noch fehlenden $20 \mathrm{~Pa}$ tienten das Studienergebnis kaum noch wesentlich beeinflusst hätten.

\section{Kommentar}

Es handelt sich um die bisher größte randomisierte und kontrollierte Studie zur Frage, ob der frühzeitige Beginn einer ART kurz nach der Infektion den Krankheitsverlauf und die Ausbildung eines Immundefekt günstig beeinflussen kann. Ob die frühzeitige Therapie tatsächlich die langfristige Prognose der HIV-Infektion beeinflusst, ist allerdings vorläufig noch nicht klar. Gegen den frühzeitigen Beginn einer ART sprechen zum Beispiel langfristige Beobachtungen, bei denen nur 27\% der Betroffenen im Zeitraum bis zwei Jahre nach der Serokonversion einen Abfall der CD4-Zellzahl < 350/ $\mu$ l erlebten. In der vorliegenden Studie war dies bereits nach 72 Wochen bei 50\% der Patienten der Fall. Offensichtlich besteht eine große Variationsbreite der Reaktion des Immunsystems auf den Erreger. Mit der frühzeitigen Therapie wird man eine Reihe von Patienten behandeln, welche diese nicht nötig haben. Ob man ihnen damit nützt oder schadet, muss vorläufig offen bleiben. H. S. FüESSL -

\section{- C. M. Hogan et al.}

The Setpoint Study (ACTG A5217): Effect of Immediate Versus Deferred Antiretroviral Therapy on Virologic Set Point in Recently HIV-1-Infected Individuals. J. Infecti. Dis. 2012; 205:87-96

\title{
HIV und HCV: indirekte Fibrosemarker prädiktiv für Mortalität
}

\section{Zwei einfach zu bestimmende Laborindices aus Transaminasen und Plättchenzahl bilden bei HCV/ HIV-koinfizierten Frauen einen unab- hängigen Prognoseparameter für die Gesamtmortalität.}

— Im Vergleich zu Personen mit HCVMonoinfektion wird bei mit HIV koinfizierten Personen in der Regel ein rasches Fortschreiten der Lebererkrankung beobachtet. Durch das längere Überleben von HIV-infizierten Patienten durch HAART sind Ärzte immer häufiger mit Leberzirrhosen und hepatozellulären Karzinomen bei ihren HIV-infizierten Patienten konfrontiert. Tatsächlich sind Lebererkrankungen derzeit die führende Todesursache von HCV/HIV koinfizierten Patienten. Über prädiktive Parameter der Mortalität koinfizierter Frauen ist wenig bekannt, da die meisten Daten bisher an Männern gewonnen wurden. Ein wichtiger prognostischer Faktor ist sicher das Ausmaß einer sich entwickelnden Leberfibrose.

In einer Kohortenstudie an 450 HCV/HIV koinfizierten Frauen aus der
Women's Interagency HIV Study der National Institutes of Health, von denen im Lauf eines medianen Follow-up von 6,6 Jahren 191 verstorben waren, wurden nun zwei einfach zu erhebende indirekte Fibrosemarker auf ihre prognostische Aussagekraft hin untersucht. Die Quotienten APRI und FIB-4, gebildet aus

(AST/oberer Normwert AST) $\times 100$

\begin{tabular}{|c|c|}
\hline Thromboyzen $\left(10^{3} / \mu \mathrm{l}\right)$ & \\
\hline Alter (Jahre) x AST & \\
\hline
\end{tabular}

wurden im Hinblick auf ihre Eignung als Prognoseparameter für die Mortalität untersucht.

Im Vergleich zu Frauen mit niedrigen APRI-Werten $(<0,5)$ wiesen Frauen mit APRI-Werten von $>1,5$ als Ausdruck einer schweren Fibrose eine Hazard Ratio der Gesamtmortalität von 2,78 auf. Ein ähnlicher Zusammenhang ergab sich für den FIB-4-Wert. Bei Frauen mit einem Wert von über 3,25 zeigte sich eine Hazard Ratio von 2,58 der Mortalität im Vergleich zu FIB-4-Werten $<1,5$. Diese beiden Parameter erwiesen sich als unabhängig von anderen Prognoseparametern der HIV-Infektion. In den fünf Jahren vor dem Tod stiegen sowohl der APRI als auch der FIB-4 deutlich an. Die Steilheit dieses Anstiegs war bei den Frauen, die an einer Lebererkrankung zu Tode kamen, deutlich größer als bei Todesfällen ohne Beteiligung der Leber.

\section{Kommentar}

Es handelt sich um die erste Longitudinalstudie, die nicht invasiv zu erhebende Marker der Leberfibrose ausschließlich bei koinfizierten Frauen mit HIV und HCV untersucht. Man kann nur staunen, wie selbst einfachsten Laborparametern immer noch neue Aspekte abzugewinnen sind. Nachdem diese Werte wahrscheinlich von allen mit HIV- und HCV-infizierten Patienten in kontinuierlicher ärztlicher Betreuung vorliegen, kann jeder nachprüfen, ob sich die Ergebnisse der Studie an der eigenen Klientel nachvollziehen lassen.

H. S. FÜESSL =

\section{- K. Bambha et al.}

Assessing mortality in women with hepatitis $C$ virus and HIV using indirect markers of fibrosis. AIDS 2012;26:599-607 